\title{
Predicative values of $\mathrm{C}$-reactive protein for the therapeutic effects of ulinastatin combined with somatostatin in severe acute pancreatitis and for the severity of gastrointestinal failure
}

\author{
YINJIA WANG and LI LI \\ Intensive Care Unit, The First People's Hospital of Kunming, Kunming, Yunnan 650224, P.R. China
}

Received November 2, 2017; Accepted February 2, 2018

DOI: $10.3892 /$ etm.2018.6577

\begin{abstract}
Severe acute pancreatitis (SAP) is a serious systemic disease with high mortality. Ulinastatin is a drug widely used for patients with SAP and multiple organ failure syndrome. The present study aimed to investigate the capacity of the serum C-reactive protein (CRP) levels to predict the therapeutic effects of ulinastatin combined with somatostatin as well as determine the severity of SAP. SAP patients were treated with ulinastatin combined with somatostatin and serum CRP levels were measured. The computed tomography severity index (CTSI), acute physiology and chronic health evaluation II (APACHE II) and gastrointestinal failure scores were used to determine the therapeutic effects. All patients were assigned to the effective group and the ineffective group. Receiver operating characteristic curve analysis was performed to determine the sensitivity and specificity of CRP levels in predicting the severity of SAP and patient prognosis. Logistic regression analysis was adopted to investigate the factors influencing the therapeutic effects. Prior to and after treatment, serum CRP levels in patients of the effective and ineffective groups were significantly different. After treatment, serum CRP levels in patients of the effective group exhibited a more obvious reduction. The sensitivity and specificity of serum CRP levels in predicting the therapeutic effects of ulinastatin combined with somatostatin in SAP patients upon hospital admission were 0.813 and 0.934 , respectively. Serum CRP levels were positively correlated with APACHE II, CTSI and gastrointestinal failure scores of SAP patients. The logistic regression demonstrated that serum albumin, creatinine and CRP levels on admission were factors influencing the therapeutic effects of ulinastatin combined with somatostatin in SAP patients. These results indicate that serum CRP levels may have a predictive value regarding the therapeutic effects
\end{abstract}

Correspondence to: $\mathrm{Dr} \mathrm{Li} \mathrm{Li}$, Intensive Care Unit, The First People's Hospital of Kunming, 504 Qingnian Road, Kunming, Yunnan 650224, P.R. China

E-mail: 1ilikm26@163.com

Key words: C-reactive protein, severe acute pancreatitis, ulinastatin, somatostatin, therapeutic effects, gastrointestinal function of ulinastatin combined with somatostatin and are an indicator of the severity of gastrointestinal failure in SAP.

\section{Introduction}

Acute pancreatitis (AP) is a common inflammatory disorder of the pancreas that may involve surrounding tissues or remote organs and lead to considerable morbidity; it has a mortality rate of $\sim 5 \%$ (1). In the US, AP is the leading cause of gastrointestinal disease associated with hospital admission and accounts for $\sim 0.27$ million hospital admissions per year and $>2.5$ billion dollars in medical expenses (2,3). Upon the establishment of the diagnosis of AP, the severity is determined according to the 2012 revision of the Atlanta classification of acute pancreatitis as follows: Severe, moderately severe or mild (4). An estimated 20-30\% of AP cases progress to severe acute pancreatitis (SAP), which rapidly progresses to include local complications and multiple organ failure (5). The mortality rate associated with SAP is $\leq 20-30 \%$ in spite of diagnostic and therapeutic advances, and there is a great requirement for emergency resuscitation and supportive treatment (6). The antiprotease drug ulinastatin has been demonstrated to be a critical therapeutic medicine for the clinical management of AP in China and Japan (7,8). Previous studies have reported the predictive role of $\mathrm{C}$-reactive protein (CRP) in pancreatitis as a biochemical marker, and the sensitivity and specificity for predicting SAP may be 80 and $76 \%$, respectively $(9,10)$.

CRP belongs to the pentraxin family of proteins with a hepatic origin, and it serves as a major component of any inflammatory reaction (11). In addition, CRP is an acute-phase protein that demonstrates a rapid increase in plasma concentration when responding to infection, acute inflammation and tissue damage (12). Specifically, CRP is secreted in response to the pro-inflammatory cytokines, including interleukin (IL)-6 and tumor necrosis factor $\alpha$ (TNF- $\alpha$ ), and it is implicated in innate immunity by facilitating phagocytosis of damaged and foreign cells, and activation of the complement pathway (13). CRP has been highlighted as a factor with prognostic significance for pancreatitis and a factor involved in the severity score for the management of AP (14). Of note, CRP levels have been indicated to be associated with the progression of SAP and have predictive value regarding mortality associated with multiple organ failure (15). Although multiple studies have focused on searching for novel biomarkers with 
better predictive value for SAP, none of them identified a superior marker to CRP (16). Therefore, the present study was performed with the major objective of exploring the serum CRP levels in SAP patients and its value in determining the severity of gastrointestinal failure, as well as predicting the therapeutic effects of ulinastatin combined with somatostatin.

\section{Materials and methods}

Study subjects. From June 2011 to June 2015, a total of 260 patients diagnosed with SAP were recruited at The First People's Hospital of Kunming (Kunming, China). A total of 172 males and 88 females (age, 22-77 years; mean age, $44.54 \pm 12.96$ years; median age, 44.5 years) were enrolled. The cohort comprised 108 patients with acute alcoholic pancreatitis, 116 patients with acute biliary pancreatitis and 36 patients with unknown causes. All patients had clinical symptoms, including paroxysmal abdominal pain, nausea, fever and vomiting, which are the diagnostic criteria for SAP according to the guidelines for diagnosis and treatment of SAP published by the Chinese Society of Gastroenterology and the Chinese Medical Association (17). Patients were included if they met at least two of the following three criteria: i) Patients with upper abdominal pain and serum amylase levels that increased to more than 3 times the normal limit; ii) computed tomography (CT) and magnetic resonance imaging (MRI) results indicating changes in the patient's condition consistent with extensive pancreatic necrosis and a Ranson's score of $>3$; and iii) patients with organ failure and with an acute physiology and chronic health evaluation II (APACHE II) score of $>8$. Patients were excluded if they met the following criteria: i) Age, $<18$ or $>80$ years; ii) patients who had acute circulatory failure, were treated with high doses of vasoactive drugs and had arterial blood lactate of $>4 \mathrm{mmol} / \mathrm{l}$; iii) triglyceride (TG) levels $>8 \mathrm{mmol} / \mathrm{l}$; iv) patients who were pregnant, had diseases of the immune system or were treated with immune enhancers or immunosuppressive agents; and v) patients who were affected by other inflammatory reactions or were treated with immunomodulatory drugs, and patients who underwent associated treatment, including bedside blood purification and thymic peptide.

Treatment regimens. All patients were subjected to conventional therapy, including oxygen inhalation, gastrointestinal decompression, fasting, supplemental plasma and nutritional support. In addition, patients were treated with $3 \mathrm{mg}$ somatostatin (batch no., 10112307; Chengdu Tiantaishan Pharmaceutical Co., Ltd., Chengdu, China) and ulinastatin (10x10 U; batch no., 08120805; Techpool Bio-pharma Co., Ltd., Guangzhou, China) in $250 \mathrm{ml} 0.9 \%$ sodium chloride for 10 days via intravenous drip (ter in die).

Observation indicators and therapeutic effect evaluation. Venous blood samples (5 $\mathrm{ml}$ each time) were separately obtained from patients on admission, days 1,3 and 7, and after treatment. Samples were collected in an anticoagulant tubes and centrifuged at $1,006 \mathrm{xg}$ for $10 \mathrm{~min}$ at $4^{\circ} \mathrm{C}$. After the serum was isolated, the samples were stored at $-65^{\circ} \mathrm{C}$. Serum CRP levels were measured using the immunoturbidimetry method with kits from Roche Diagnostics (Basel, Switzerland) in
Table I. Gastrointestinal Failure score in patients with severe acute pancreatitis.

Clinical symptomatology

Score

Normal gastrointestinal function

0

Enteral feeding $<50 \%$ of calculated needs or no

feeding 3 days after abdominal surgery

Food intolerance (enteral feeding not applicable due

2

to high gastric aspirate volume, vomiting, bowel

distension, or severe diarrhoea) or IAH

Food intolerance and IAH

3

Abdominal compartment syndrome

4

IAH, intra-abdominal hypertension.

accordance with the manufacturer's protocol. A therapeutic effects evaluation was performed according to the guidelines for the diagnosis and treatment of SAP published by the Chinese Society of Gastroenterology and the Chinese Medical Association (17). The outcomes were defined as follows: i) Cured, the patient's clinical symptoms completely disappeared and CT scan results were normal; ii) markedly effective, the patient's clinical symptoms improved significantly and CT scan results were normal; iii) effective, the patient's clinical symptoms improved to a certain extent and the amylase in the patient's hematuria was markedly reduced, but the CT scan still exhibited certain some abnormalities; iv) ineffective, the patient's clinical symptoms were not alleviated and CT scan results revealed no obvious improvement. The total efficiency was calculated from the number of respective cases (n) as follows: Total efficiency $=\left(\mathrm{n}_{\text {cured }}+\mathrm{n}_{\text {markedly effective }}+\mathrm{n}_{\text {effective }}\right) / \mathrm{n}_{\text {total }}$ $\mathrm{x} 100 \%$. APACHE II and computer tomography severity index (CTSI) scores were determined to assess the patients. Each patient underwent an enhanced CT scan on admission, and Balthazar grading and CTSI scores were determined (18). Indexes were determined as follows: Balthazar grades D \& E were denoted as 1 and 2 points; furthermore, necrosis was scored as follows: No necrosis, $0 ;>33 \%$ necrosis, $2 ; 33-50 \%$ necrosis, 4 ; and $>50 \%$ necrosis, 6 . Addition of the two scores resulted in the CTSI score (range, 7-9 points). The criteria for the gastrointestinal failure score were based on a previous study (19). The gastrointestinal failure score was graded as Table I.

Statistical analysis. Statistical analyses were performed using SPSS 21.0 (IBM Corp., Armonk, NY, USA) and GraphPad Prism (GraphPad Software Inc., La Jolla, CA, USA). Values are expressed as the mean \pm standard deviation. Differences between two groups were compared using Student's t-test. Enumeration data are expressed as $\mathrm{n}$ or a ratio, and the differences between groups were analyzed using the chi-square test. Receiver operating characteristic curve (ROC) analysis was performed to determine the sensitivity and specificity of CRP levels in predicting the severity of SAP and patient prognosis. Spearman's rank correlation coefficient was determined to assess the correlation of associated factors. Logistic regression analysis was used to investigate the influencing factors 
Table II. Baseline characteristics of patients with severe acute pancreatitis.

\begin{tabular}{|c|c|c|c|}
\hline Baseline characteristics & Effective group $(n=244)$ & Ineffective group $(n=16)$ & P-value \\
\hline Age (years) & $43.84 \pm 12.50$ & $55.23 \pm 15.42$ & 0.001 \\
\hline Sex & & & 0.589 \\
\hline Male & $126(51.64 \%)$ & $10(62.5 \%)$ & \\
\hline Female & $118(48.36 \%)$ & $6(37.5 \%)$ & \\
\hline Ascites & & & 0.782 \\
\hline No & $168(68.85 \%)$ & $12(75 \%)$ & \\
\hline Yes & $76(31.15 \%)$ & $4(25 \%)$ & \\
\hline Body temperature $\left({ }^{\circ} \mathrm{C}\right)$ & $36.6 \pm 1.6$ & $36.9 \pm 0.8$ & 0.458 \\
\hline Heart rate $(\mathrm{bpm})$ & $115.4 \pm 19.7$ & $119.8 \pm 21.3$ & 0.390 \\
\hline Mean arterial pressure $(\mathrm{mmHg})$ & $97.4 \pm 19.0$ & $92.8 \pm 17.8$ & 0.347 \\
\hline 24-h urine volume $(\mathrm{ml})$ & $1389.12 \pm 625.45$ & $1078.10 \pm 642.36$ & 0.056 \\
\hline CTSI score & $5.34 \pm 1.89$ & $6.78 \pm 2.24$ & 0.004 \\
\hline Gastrointestinal failure score & $2.13 \pm 0.45$ & $3.67 \pm 0.30$ & $<0.001$ \\
\hline APACHE II score & $8.56 \pm 4.36$ & $12.24 \pm 6.02$ & 0.002 \\
\hline White blood cells (x109/1) & $17.0 \pm 12.1$ & $19.1 \pm 9.0$ & 0.742 \\
\hline Rate of neutrophils (\%) & $82.25 \pm 6.74$ & $83.81 \pm 11.72$ & 0.397 \\
\hline Hematocrit (\%) & $0.39 \pm 0.06$ & $0.39 \pm 0.07$ & $>0.999$ \\
\hline Serum albumin $(\mathrm{g} / \mathrm{l})$ & $28.9 \pm 4.5$ & $25.3 \pm 4.2$ & 0.002 \\
\hline Urea nitrogen $(\mathrm{mmol} / \mathrm{l})$ & $6.47 \pm 3.04$ & $15.90 \pm 6.8$ & $<0.001$ \\
\hline Serum creatinine $(\mu \mathrm{mol} / \mathrm{l})$ & $96.4 \pm 51.2$ & $136.9 \pm 16.4$ & 0.002 \\
\hline Blood glucose (mmol/l) & $10.42 \pm 4.26$ & $12.33 \pm 8.45$ & 0.077 \\
\hline Triglycerides (mmol/l) & $7.35 \pm 7.70$ & $5.26 \pm 6.34$ & 0.289 \\
\hline Blood calcium (mmol/l) & $1.78 \pm 0.30$ & $1.68 \pm 0.42$ & 0.210 \\
\hline Serum amylase (U/1) & $624.32 \pm 472.54$ & $846.26 \pm 1191.82$ & 0.084 \\
\hline APTT (sec) & $35.89 \pm 13.25$ & $27.99 \pm 20.16$ & 0.053 \\
\hline Arterial partial pressure of oxygen $(\mathrm{mmHg})$ & $71.36 \pm 12.02$ & $66.32 \pm 20.26$ & 0.124 \\
\hline Blood $\mathrm{pH}$ & $7.33 \pm 0.07$ & $7.29 \pm 0.18$ & 0.056 \\
\hline Urinary albumin & & & 0.102 \\
\hline Negative & 73 & 8 & \\
\hline Positive & 171 & 8 & \\
\hline
\end{tabular}

CTSI, computed tomography severity index; APACHE II, acute physiology and chronic health evaluation II; APTT, activated partial thromboplastin time. Values are expressed as the mean \pm standard deviation. Enumeration data are expressed as $n$ or a ratio.

of the therapeutic effects. $\mathrm{P}<0.05$ was considered to indicate a statistically significant difference.

\section{Results}

Baseline characteristics. The SAP patients $(n=260)$ were treated with ulinastatin combined with somatostatin. At the end of the treatment, the outcome was as follows: 4 cases of peripancreatic infection, 8 cases of multiple organ failure, 8 cases of pseudocyst, 63 cured cases, and 155 cases with markedly effective, 26 cases with effective and 16 with ineffective treatment. The total effective rate was $93.8 \%$. The patients were assigned to an effective group $(n=244)$ and an ineffective group ( $n=16)$ according to the clinical effect. The effective group comprised 126 males and 118 females and the ineffective group comprised 10 males and 6 females. Significant differences were identified in age, CTSI score, APACHE II score, gastrointestinal failure score, serum albumin levels, blood urea nitrogen (BUN) and serum creatinine levels between the two groups $(\mathrm{P}<0.05)$. There were no significant differences in gender, heart rate on admission, mean arterial pressure, 24-h urine volume, white blood cell count, the rate of neutrophil count, blood glucose, triglycerides, blood calcium, serum amylase, activated partial thromboplastin time, arterial partial pressure of oxygen, blood $\mathrm{pH}$ and urinary albumin ( $\mathrm{P}>0.05$; Table II).

Lower serum CRP levels are identified in SAP patients with effective treatment by ulinastatin combined with somatostatin. Immunoturbidimetry was performed to determine the serum CRP levels of patients. As presented in Fig. 1, prior to treatment, serum CRP levels in the patients of the effective group were $157.54 \pm 15.02 \mathrm{mg} / \mathrm{l}$, and serum CRP levels in the patients of the ineffective group were $233.58 \pm 18.85 \mathrm{mg} / \mathrm{l}$. The 


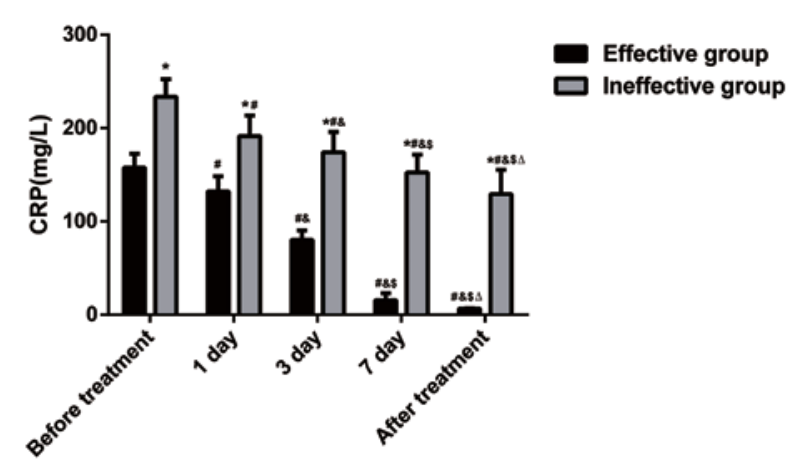

Figure 1. Serum CRP levels in patients of the effective group and the ineffective group. CRP, $C$-reactive protein; ${ }^{*} \mathrm{P}<0.001$ vs. the effective group; ${ }^{*} \mathrm{P}<0.001$ vs. CRP levels prior to treatment; ${ }^{\circledR} \mathrm{P}<0.001$ vs. CRP levels following 1 day of treatment; ${ }^{~} \mathrm{P}<0.001$ vs. CRP levels following 3 days of treatment; ${ }^{\Delta} \mathrm{P}<0.001$ vs. CRP levels following 7 days of treatment.

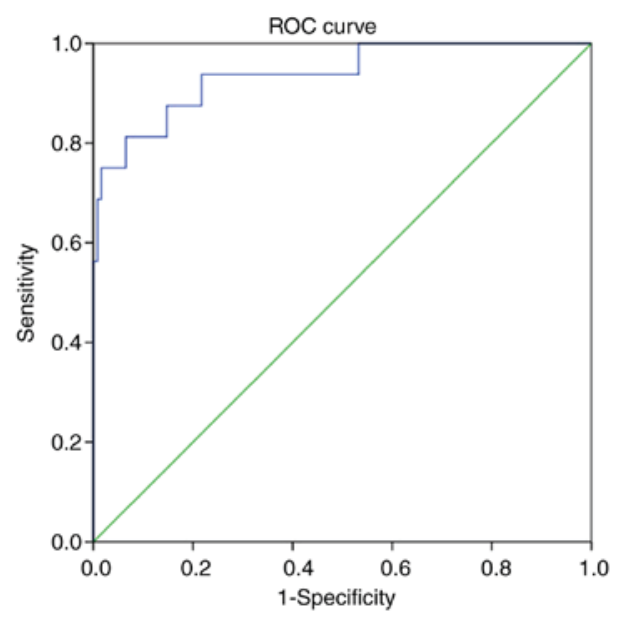

Figure 2. ROC curve for determining the sensitivity and specificity of serum CRP levels on admission for the prediction of the therapeutic effects in patients with severe acute pancreatitis. ROC curve, receiver operating characteristic curve; CRP, C-reactive protein.

difference between the two groups was statistically significant $(\mathrm{P}<0.05)$. After treatment, serum CRP levels in patients of the two groups exhibited a downward trend, but serum CRP levels were more significantly decreased in the effective group. The CRP levels between the two groups were significantly different at days 1,3 and 7 of treatment $(\mathrm{P}<0.05)$. The serum CRP levels in patients of the effective group were $6.56 \pm 0.22 \mathrm{mg} / \mathrm{l}$ after treatment, and those in patients of the ineffective group were $129.28 \pm 36.21 \mathrm{mg} / \mathrm{l}$ after treatment; the difference was significant between the two groups $(\mathrm{P}<0.05)$.

Serum CRP levels have the predictive value for the therapeutic effects of ulinastatin combined with somatostatin in SAP. Analysis of the ROC curve for the prediction of the therapeutic effects of ulinastatin combined with somatostatin based on the CRP provided an area under the curve of $0.938[\mathrm{P}<0.001 ; 95 \%$ confidence interval, 0.870-1.000]. With the optimal threshold of $177.17 \mathrm{mg} / \mathrm{l}$, the sensitivity and specificity were 0.813 and 0.934 , respectively. The results indicated that serum CRP levels had a high predictive value for the therapeutic effects of ulinastatin combined with somatostatin in SAP (Fig. 2).
Correlation between serum CRP levels and gastrointestinal failure in SAP patients. Correlation analyses of serum CRP levels, CTSI score, APACHE II score, and gastrointestinal failure score in patients on admission are presented in Fig. 3. The analysis revealed that serum CRP levels were significantly and positively correlated with the APACHE II score (Spearman's rank correlation coefficient $=0.674 ; \mathrm{P}<0.001$; Fig. 3A). The analysis of serum CRP levels and the CTSI score demonstrated that higher serum CRP levels corresponded with a more severe condition expressed as a higher CTSI score (Spearman's rank correlation coefficient $=0.707 ; \mathrm{P}<0.001$; Fig. 3B). In addition, the correlation analysis indicated that serum CRP levels directly reflected the gastrointestinal failure of SAP patients (Spearman's rank correlation coefficient=0.736; $\mathrm{P}<0.001$; Fig. 3C).

Serum albumin, creatinine and CRP levels are factors affecting the therapeutic effects of ulinastatin combined with somatostatin in SAP patients. For the logistic regression analysis (Table III), the therapeutic effect of ulinastatin combined with somatostatin was regarded as the dependent variable, and gender, CTSI score, APACHE II score, gastrointestinal failure score, serum albumin levels, BUN, serum creatinine and serum CRP levels on admission were considered as independent variables. The results indicated that serum albumin, serum creatinine and serum CRP levels on admission were significantly associated with the therapeutic effects of ulinastatin combined with somatostatin in SAP patients $(\mathrm{P}<0.05)$. However, gender, CTSI score, APACHE II score, gastrointestinal failure score and BUN were not correlated with the therapeutic effects of ulinastatin combined with somatostatin in SAP patients $(\mathrm{P}>0.05)$.

\section{Discussion}

SAP is an acute disease of the gastrointestinal tract that leads to intense systemic inflammatory responses and progresses quickly from local pancreatic impairment to multiple organ failure (5). Ulinastatin and somatostatin are widely used in the treatment of AP, but the therapeutic effects and the mechanism of how they function have not been clearly established $(20,21)$. In addition, multiple studies have reported on the diagnostic and predictive role of CRP in pancreatitis $(22,23)$. Therefore, the present study was performed with the central aim of exploring the role of serum CRP levels in predicting the therapeutic effects of ulinastatin combined with somatostatin in the treatment of SAP and determining the severity of gastrointestinal failure.

Prior to and after treatment, serum CRP levels were significantly lower in patients with effective and ineffective treatment outcomes; however, those with an effective treatment outcome had a more obvious reduction after treatment. The sensitivity and specificity of serum CRP levels in predicting the therapeutic effects of ulinastatin combined with somatostatin in SAP patients upon hospital admission were 0.813 and 0.934 , respectively. These results indicated that serum levels of CRP may serve as a predictive indicator for the therapeutic effects of ulinastatin combined with somatostatin in SAP. The results of a previous study corroborated with the present results, suggesting that the level of CRP is a predictor of the 
Table III. Logistic regression analysis of factors associated with the therapeutic efficacy in patients with severe acute pancreatitis.

\begin{tabular}{lccccrrr}
\hline Factor & B & S.E. & Wald & df & P-value & OR & 95\% CI \\
\hline Age & 0.001 & 0.028 & 0 & 1 & 0.982 & 1.001 & $0.948-1.056$ \\
CTSI score & -0.066 & 0.155 & 0.179 & 1 & 0.672 & 0.936 & $0.691-1.269$ \\
APACHEII score & 0.027 & 0.077 & 0.124 & 1 & 0.725 & 1.027 & $0.884-1.194$ \\
Gastrointestinal failure score & 0.375 & 0.403 & 0.896 & 1 & 0.354 & 1.294 & $0.721-2.322$ \\
Serum albumin & -0.643 & 0.200 & 10.316 & 1 & 0.001 & 0.526 & $0.355-0.778$ \\
Blood urea nitrogen & 0.187 & 0.191 & 0.961 & 1 & 0.327 & 1.206 & $0.830-1.752$ \\
Serum creatinine & -0.099 & 0.023 & 18.018 & 1 & $<0.001$ & 0.906 & $0.866-0.948$ \\
Serum CRP levels on admission & 0.053 & 0.027 & 3.952 & 1 & 0.047 & 1.055 & $1.001-1.112$
\end{tabular}

CTSI, computed tomography severity index; APACHE II, acute physiology and chronic health evaluation II; CRP, C-reactive protein; B, partial regression coefficient; S.E., standard error; df, degree of freedom, OR, odds ratio; 95\% CI, 95\% confidence interval.
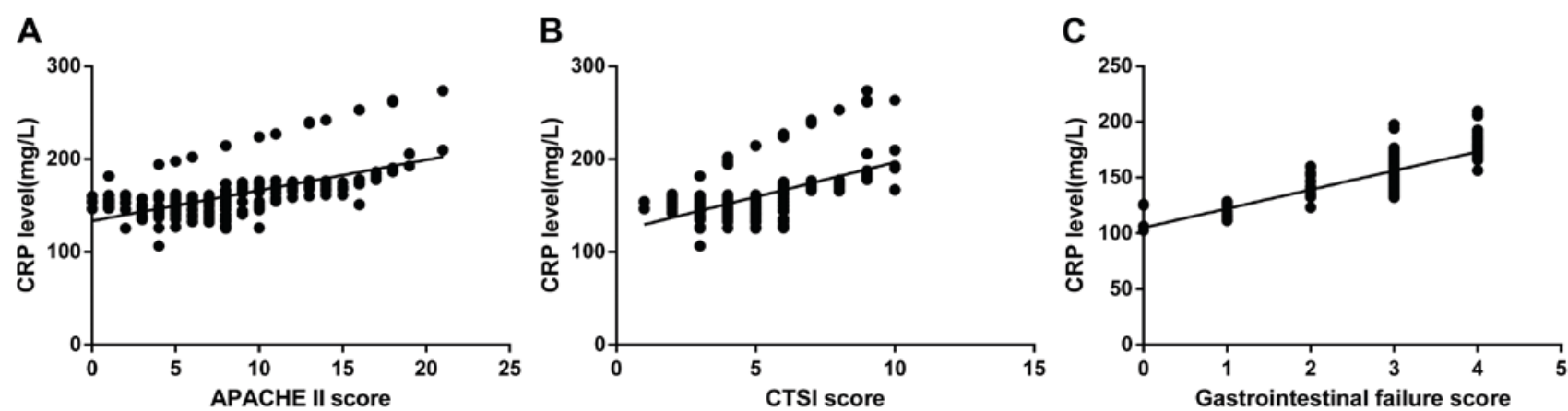

Figure 3. Correlation analyses of serum CRP levels, CTSI score, APACHE II score and gastrointestinal failure score in patients with severe acute pancreatitis on admission. (A) Correlation between serum CRP levels and APACHE II score; (B) correlation between serum CRP levels and CTSI score; (C) correlation between serum CRP levels and gastrointestinal failure score. CRP, C-reactive protein; CTSI, computed tomography severity index; APACHE II, acute physiology and chronic health evaluation II.

severity of SAP (9). In the study of Schütte et al (24), CRP was proposed as a single reference parameter of pancreatic necrosis, as it may be determined via a readily available and inexpensive laboratory test and has good prognostic value in the clinical setting. In the treatment of AP, ulinastatin suppresses pancreatic activity or ameliorates the severity of pancreatic injury by exerting anti-inflammatory effects through reducing serum levels of inflammatory cytokines, including CRP, IL-6 and TNF- $\alpha$ (25). Furthermore, CRP, as a well-documented biomarker for predicting SAP, has a crucial pathophysiological role in pancreatitis, is linked with the associated morbidity and mortality, and is positively correlated with clinical end-points in AP patients $(22,26)$. Consistent with the present study, Cardoso et al (27) performed a population-based cohort study to determine clinical features based on the prescription of prophylactic antibiotics for AP patients. They revealed that the elevation of serum CRP levels contributed to clinicians' decisions to administer prophylactic antibiotics. The present study further substantiated the results that CRP has a predictive role regarding the therapeutic effects of ulinastatin combined with somatostatin in SAP and may be an indicator of gastrointestinal failure severity in SAP.

In the present study, serum CRP levels were positively correlated with APACHE II, CTSI and gastrointestinal failure scores of SAP patients. The logistic regression analysis demonstrated that serum albumin, creatinine and CRP levels on admission were factors influencing the therapeutic effects of ulinastatin combined with somatostatin in SAP patients. Ulinastatin enables the stabilization of lysosomal membranes, and suppresses trypsin and the release of inflammatory cytokines, thereby repressing organ failure and other serious illnesses (28). SAP compromises immune function, whereas ulinastatin restores immune function by enhancing the cytokine release of splenocytes and proliferation responses (8). Based on the results of the present correlation analysis, it may be concluded that serum CRP levels directly reflect the gastrointestinal function of SAP patients. The major cause of intestinal mucosal injury during AP has been reported to be the excessive secretion of inflammatory mediators (29). A possible mechanism involved is that of somatostatin, which suppresses the production of pancreatic enzymes and represses the motor activity of Oddi's sphincter and Oddi basal pressure, while protecting gastrointestinal mucosal cells and stimulating the reticulo-endothelial system (30). Furthermore, patients with Crohn's disease, a chronic inflammatory bowel disease, who have increased serum CRP levels, demonstrate more active inflammation are more susceptible to a high clinical efficacy of infliximab (anti-TNF- $\alpha$ monoclonal antibody) when compared with those patients with low serum CRP levels (31). According to a study of Tachyla et al (15), the elevated CRP 
levels indicate the progression of systemic inflammatory responses, which are correlated with the progression of multiple organ failure. According to the logistic regression analysis, serum albumin, creatinine and CRP levels are factors affecting the therapeutic effects of ulinastatin combined with somatostatin in SAP patients, which is consistent with previous studies $(32,33)$.

In conclusion, the results of the present study indicated that lower serum CRP levels prior to and after treatment led to better therapeutic effects of ulinastatin combined with somatostatin in SAP patients. Furthermore, a positive correlation was determined between serum CRP levels and the severity of gastrointestinal failure. Accordingly, serum CRP levels may serve as a predictive indicator for the therapeutic effects of ulinastatin combined with somatostatin and the severity of gastrointestinal failure in SAP. In addition, a better understanding of the association between serum CRP levels and the efficacy of ulinastatin combined with somatostatin in SAP may provide a basis for the development of novel treatment regimens to reduce pancreatic activity and ameliorate its symptoms and multiple organ failure. Due to a limited sample size and the experimental time window, an extended investigation is recommended in the future.

\section{Acknowledgements}

Not applicable.

\section{Funding}

No funding was received.

\section{Availability of data and materials}

The analyzed data sets generated during the study are available from the corresponding author on reasonable request.

\section{Authors' contributions}

LL conceived, designed and developed all methodology. YW Acquired data (acquired and managed patients and provided facilities), analyzed results and interpreted data. YW and LL wrote, reviewed and revised the manuscript. The final version of the manuscript has been read and approved by all authors, and each author believes that the manuscript represents honest work.

\section{Ethical approval and consent to participate}

The study was approved by the Institutional Review Board of The First People's Hospital of Kunming. All participants signed a document of informed consent.

\section{Patient consent for publication}

Not applicable.

\section{Competing interests}

The authors declare that they have no competing interests.

\section{References}

1. Türkvatan A, Erden A, Türkoğlu MA, Seçil M and Yener Ö: Imaging of acute pancreatitis and its complications. Part 1: Acute pancreatitis. Diagn Interv Imaging 96: 151-160, 2015.

2. Kuramatsu JB, Huttner HB and Schwab S: Advances in the management of intracerebral hemorrhage. J Neural Transm (Vienna) 120 (Suppl 1): S35-S41, 2013.

3. Yadav D and Lowenfels AB: The epidemiology of pancreatitis and pancreatic cancer. Gastroenterology 144: 1252-1261, 2013.

4. Maheshwari R and Subramanian RM: Severe acute pancreatitis and necrotizing pancreatitis. Crit Care Clin 32: 279-290, 2016.

5. Yang ZW, Meng XX and Xu P: Central role of neutrophil in the pathogenesis of severe acute pancreatitis. J Cell Mol Med 19: 2513-2520, 2015.

6. Sternby H, Hartman H, Johansen D, Thorlacius H and Regner S: Predictive capacity of biomarkers for severe acute pancreatitis. Eur Surg Res 56: 154-163, 2016.

7. Liu R, Qi H, Wang J, Wang Y, Cui L, Wen Y and Yin C: Ulinastatin activates the renin-angiotensin system to ameliorate the pathophysiology of severe acute pancreatitis. J Gastroenterol Hepatol 29: 1328-1337, 2014.

8. Chen S, Shi H, Zou X and Luo H: Role of ulinastatin in preventing post-endoscopic retrograde cholangiopancreatography pancreatitis: The Emperor's new clothes or aladdin's magic lamp? Pancreas 39: 1231-1237, 2010.

9. Jia R, Tang M, Qiu L, Sun R, Cheng L, Ma X, Yin G, Hu G, Wang $X$ and Zhao Y: Increased interleukin-23/17 axis and C-reactive protein are associated with severity of acute pancreatitis in patients. Pancreas 44: 321-325, 2015.

10. Gurleyik G, Emir S, Kilicoglu G, Arman A and Saglam A: Computed tomography severity index, APACHE II score, and serum CRP concentration for predicting the severity of acute pancreatitis. JOP 6: 562-567, 2005

11. Ansar W and Ghosh S: C-reactive protein and the biology of disease. Immunol Res 56: 131-142, 2013.

12. Allin KH and Nordestgaard BG: Elevated C-reactive protein in the diagnosis, prognosis, and cause of cancer. Crit Rev Clin Lab Sci 48: 155-170, 2011

13. Singh PP, Zeng IS, Srinivasa S, Lemanu DP, Connolly AB and Hill AG: Systematic review and meta-analysis of use of serum C-reactive protein levels to predict anastomotic leak after colorectal surgery. Br J Surg 101: 339-346, 2014.

14. Sato T, Ohno K, Tamamoto T, Oishi M, Kanemoto H, Fukushima K, Goto-Koshino Y, Takahashi M and Tsujimoto H: Assesment of severity and changes in C-reactive protein concentration and various biomarkers in dogs with pancreatitis. J Vet Med Sci 79: 35-40, 2017.

15. Tachyla SA, Marochkov AV, Lipnitski AL and Nikiforova YG: The prognostic value of procalcitonin, $\mathrm{C}$-reactive protein and cholesterol in patients with an infection and multiple organ dysfunction. Korean J Anesthesiol 70: 305-310, 2017.

16. Pongprasobchai S, Jianjaroonwong V, Charatcharoenwitthaya $\mathrm{P}$, Komoltri C, Tanwandee T, Leelakusolvong S, Pausawasdi N, Srikureja W, Chainuvati S, Prachayakul V, et al: Erythrocyte sedimentation rate and C-reactive protein for the prediction of severity of acute pancreatitis. Pancreas 39: 1226-1230, 2010.

17. Loveday BP, Srinivasa S, Vather R, Mittal A, Petrov MS, Phillips AR and Windsor JA: High quantity and variable quality of guidelines for acute pancreatitis: A systematic review. Am J Gastroenterol 105: 1466-1476, 2010.

18. Tsuji Y, Takahashi N and Tsutomu C: Pancreatic perfusion CT in early stage of severe acute pancreatitis. Int J Inflam 2012: 497386, 2012.

19. Reintam A, Parm P, Kitus R, Starkopf J and Kern H: Gastrointestinal failure score in critically ill patients: A prospective observational study. Crit Care 12: R90, 2008.

20. Wang G, Wen J, Wilbur RR, Wen P, Zhou SF and Xiao X: The effect of somatostatin, ulinastatin and Salvia miltiorrhiza on severe acute pancreatitis treatment. Am J Med Sci 346: 371-376, 2013.

21. Yoo YW, Cha SW, Kim A, Na SY, Lee YW, Kim SH, Lee HIe, Lee YJ, Yang HW and Jung SH: The use of gabexate mesylate and ulinastatin for the prevention of post-endoscopic retrograde cholangiopancreatography pancreatitis. Gut Liver 6: 256-261, 2012.

22. Yin G, Hu G, Cang X, Yu G, Hu Y, Xing M, Chen C, Huang Y, Tang M, Zhao Y, et al: C-reactive protein: Rethinking its role in evaluating the severity of hyperlipidemic acute pancreatitis. Pancreas 43: 1323-1328, 2014. 
23. Mroczko B, Groblewska M, Gryko M, Kedra B and Szmitkowski M: Diagnostic usefulness of serum interleukin 6 (IL-6) and C-reactive protein (CRP) in the differentiation between pancreatic cancer and chronic pancreatitis. J Clin Lab Anal 24: 256-261, 2010

24. Schütte K and Malfertheiner P: Markers for predicting severity and progression of acute pancreatitis. Best Pract Res Clin Gastroenterol 22: 75-90, 2008

25. Zhang C, Wang Y, Fu W, Zhang W, Wang T and Qin H: A Meta-analysis on the effect of ulinastatin on serum levels of C-reactive protein, interleukin 6, and tumor necrosis factor alpha in Asian patients with acute pancreatitis. Genet Test Mol Biomarkers 20: 118-124, 2016.

26. Vermeire S, Van Assche G and Rutgeerts P: The role of $\mathrm{C}$-reactive protein as an inflammatory marker in gastrointestinal diseases. Nat Clin Pract Gastroenterol Hepatol 2: 580-586, 2005.

27. Cardoso FS, Ricardo L, Gondar P, Deus JR and Horta D: C-reactive protein may influence decisively the prescription of prophylactic antibiotics in acute pancreatitis: A population-based cohort study. Pancreas 44: 404-408, 2015.
28. Wang X, Zhuang X, Wei R, Wang C, Xue X and Mao L: Protective effects of Acanthopanax vs. Ulinastatin against severe acute pancreatitis-induced brain injury in rats. Int Immunopharmacol 24: 285-298, 2015.

29. Shen Y,CuiN,MiaoB andZhaoE:Immune dysregulationin patients with severe acute pancreatitis. Inflammation 34: 36-42, 2011.

30. Wang G, Liu Y, Zhou SF, Qiu P, Xu L, Wen P, Wen J and Xiao X: Effect of Somatostatin, Ulinastatin and gabexate on the treatment of severe acute pancreatitis. Am J Med Sci 351: 506-512, 2016.

31. Reinisch W, Wang Y, Oddens BJ and Link R: C-reactive protein, an indicator for maintained response or remission to infliximab in patients with Crohn's disease: A post-hoc analysis from ACCENT I. Aliment Pharmacol Ther 35: 568-576, 2012.

32. Muddana V, Whitcomb DC, Khalid A, Slivka A and Papachristou GI: Elevated serum creatinine as a marker of pancreatic necrosis in acute pancreatitis. Am J Gastroenterol 104: 164-170, 2009.

33. Chen Y, Zhang ZW, Wang B, Yin WH, Zuo YY, Kang Y and Liu J: Relationship between early serum albumin variation and prognosis in patients with severe acute pancreatitis treated in ICU. Sichuan Da Xue Xue Bao Yi Xue Ban 44: 237-241, 2013 (In Chinese). 